\title{
Perspectives on Pharmacological Prevention of Pacing Induced Heart Remodeling: Targeting Extracellular Matrix
}

\author{
Andrzej Tomasik* \\ Department of Cardiology in Zabrze, Medical University of Silesia in Katowice UI. Skłodowskiej 10, 41-800 Zabrze, Poland
}

\begin{abstract}
Right ventricle apical pacing causes dyssynchronous left ventricle contraction. Dyssynchrony leads to ventricular remodeling and an overt heart failure eventually. Experimental animal studies lend the evidence that dyssynchronous contraction is accompanied by tremendous derangement in extracellular compartment with altered collagen content, activity and expression of matrix metalloproteinases and their inhibitors. The capability of some therapeutic agents like angiotensin converting enzyme inhibitors, angiotensin receptor blockers or beta blockers to prevent postinfarction left ventricle remodeling should be considered as an anti-remodeling treatment in patients with implanted pacemakers and obligatory right ventricle pacing. We summarize the evidence and rationale for such preventive management.
\end{abstract}

Keywords: Dual chamber pacemakers; Apical pacing; Left ventricle remodeling; Heart failure, Prevention

\section{Introduction}

Permanent pacing is a life-saving and quality of life-improving therapy. However, in patients requiring obligatory pacing, as in complete atrioventricular block, significant clinical consequences might appear and turn into an overt heart failure. Several papers document significant dyssynchrony in left ventricle contraction, evidenced by tissue Doppler echocardiography $[1,2]$. Three months of atrial synchronized ventricle pacing are sufficient to cause left ventricle to enlarge, to decrease left ventricle ejection fraction, and to deteriorate its diastolic function [1]. Longer obligatory right ventricle apical pacing (at least five years long) in patients with congenital complete atrioventricular block is sufficient to reduce significantly cardiac output and to decrease the patients' exercise capacity [2]. Tops et al. [3] using speckle-tracking strain analysis, have reported significant heterogeneity in time-to-peak strain in six myocardial segments after a mean of 3.8 years of right ventricle pacing. In $57 \%$ of patients in whom left ventricle (LV) dyssynchrony, represented by a time difference $\geq 130$ ms between the time-to-peak strain of the (antero)septal and the posterolateral segments, was present a deterioration of left ventricle systolic function and New York Heart Association (NYHA) functional class was observed. Recently published papers $[4,5]$ have been focused on clinical symptoms in pacemaker recipients. De Sa et al. [4] have reported that $40 \%$ of patients have worsened NYHA functional class from class I to class II-III over a period of 24 months along with significant increase in brain natriuretic peptide (BNP) concentration. Zhang et al. [5] have reported $26 \%$ incidence of new-onset heart failure in a group of pacemaker recipients with normal ejection fraction. The new-onset heart failure was associated with approximately 12 fold higher cardiovascular mortality. The ominous sequence of detrimental effect of right ventricular apical pacing is shown in Figure 1. As it will be discussed later, the primary heart compartment involved in pacing-induced remodeling, is extracellular matrix (ECM). It organizes and supports cardiomyocytes, enables their effective contraction, and serves important metabolic and signaling function. It consists mainly of structural proteins (type I and III collagen, fibronectin, laminins) and non-structural matricellular proteins, as well as proteinase enzymes and their inhibitors.

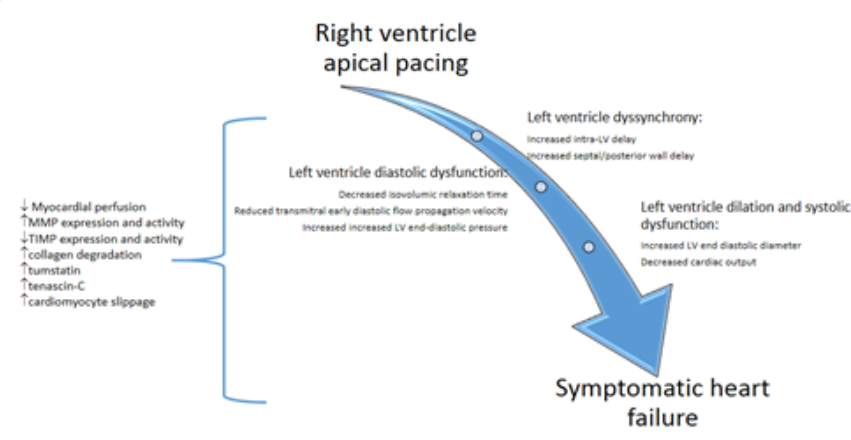

Figure 1: Detrimental effect of right ventricle apical pacing.

\section{Detrimental Effect of Pacing-Underlying Mechanisms}

There are several papers elucidating the underlying mechanisms of detrimental effect of right ventricle apical pacing [6-8]. Tse et al. [8] have proved that patients with right ventricle apical pacing have had significantly increased perfusion defects in dipyridamole thallium myocardial scintigraphy along with regional wall motion abnormalities in radionuclide ventriculography. These changes were more pronounced at 18 months after pacemaker implantation.

The animal experimental models [6,7] provide much more detailed characteristic of consequences of pacing. Both experiments were carried out in dogs, but researchers have studied different pacing sites. Garcia et al. [6] have used atrial and left ventricle epicardial pacing in

*Corresponding author: Andrzej Tomasik, Department of Cardiology in Zabrze, Medical University of Silesia in Katowice UI, Skłodowskiej 10, 41800 Zabrze, Poland, Tel: +48 3237323 72; Fax: +48 3227110 10; Email: atomasik@sum.edu.pl

Received July 28, 2015; Accepted September 08, 2015; Published September 10, 2015

Citation: Tomasik A (2015) Perspectives on Pharmacological Prevention of Pacing Induced Heart Remodeling: Targeting Extracellular Matrix. Single Cell Biol 4: 120. doi:10.4172/2168-9431.1000120

Copyright: (c) 2015 Tomasik. This is an open-access article distributed under the terms of the Creative Commons Attribution License, which permits unrestricted use, distribution, and reproduction in any medium, provided the original author and source are credited. 
an open chest anesthetized dogs, as Lin et al. [7] have used obligatory atrial synchronized right ventricle apical pacing in dogs, which have been subjected to atrioventricular node ablation. Moreover, Garcia et al. [6] have used sonomicrometric sensors to monitor dyssynchrony of ventricular contraction at the pacing and remote to pacing lead areas of left ventricle, as well as left ventricle catheter to assess left ventricle pressure curve. They have proven that pacing cause's dyskinesis at the pacing site and that even short stimulation time (less than four hours) is sufficient to induce alterations in extracellular matrix. Myocardial matrix metalloproteinase activity showed a sevenfold increase in substrate cleavage at the pacing site relative to the remote site. Gelatin zymography revealed increases in $92-\mathrm{kDa}$ matrix metalloproteinase- 9 (MMP-9) activity and 86-kDa MMP-9 activity at the pacing site relative to the remote site, whereas MMP-2 activity was unaffected. Wall motion abnormalities, recorded at pacing site, were accompanied by increased collagen degradation, plasmin activity, nitrotyrosine levels, and inflammatory infiltrate relative to the remote site.

Specific links for further elucidation of underlying mechanisms come from the results of studies on myocardial infarction. First, tenascin- $\mathrm{C}$, one of the matricellular proteins that is barely detectable in adult hearts, is re-expressed after myocardial infarction $[9,10]$. Importantly, its expression is induced by mechanical stress and it mediates de-adhesion of cell-extracellular matrix surfaces and eventually leads to cardiomyocyte slippage [11]. Secondly, matrix metalloproteinase cleavage products, known as matricryptins are potent, endogenous inhibitors of angiogenesis [12]. For example, matrix metalloproteinase- 9 acts upon collagen IV and produces tumstatin, an inhibitor of angiogenesis $[13,14]$. This may serve as simultaneous explanation for degradation of fibrillar content of ECM and myocardial perfusion defects observed by Tse et al. [8]. Although, pacing induced remodeling is a relatively long-lasting process and there is no evidence of cardiac myocyte necrosis, as observed in myocardial infarction setting, increased matrix metalloproteinase activity and its protein expression seem to play a crucial role in cardiac remodeling [15].

\section{Inhibition of Matrix Metalloproteinase Activity}

The concept of matrix metalloproteinase inhibition has led to development of an array of MMP inhibitors that were primarily directed towards cancer or rheumatoid/inflammatory diseases [1620]. PG-116800, selective, oral MMP inhibitor, based on hydroxamic acid molecule, was studied in a clinical trial PREMIER [21]. Results of the PREMIER study (Prevention of Myocardial Infarction Early Remodeling) were negative and there was increased incidence of arthralgia and joint stiffness in the active arm participants.

Spinale et al. [22] have studied a PD166793 MMP inhibitor in a pig model of rapid pacing induced heart failure. The examined MMP inhibitor did not completely prevent the degree of LV dilation with rapid pacing. Most of examined inhibitors have exhibited serious side effects with musculoskeletal syndrome described most often. Currently only one tetracycline derivative MMP inhibitor has FDA approval for periodontitis treatment.

However, the report of Sawicki et al. [23] provides an excellent evidence for efficacy of angiotensin type 1 (AT1) receptor blockade with valsartan to prevent heart remodeling in ischemia-reperfusion induced injury in dogs. Valsartan has limited LV dysfunction, infarct size, increased tissue inhibitor of matrix metalloproteinase 3 (TIMP3) and the TIMP-3/MMP-9 ratio. The beneficial effect of valsartan on decreased MMP-9 activity along with improved left ventricle remodeling in a small group of acute myocardial infarction (AMI) patients treated with primary coronary intervention was reported either [24]. These combined data on valsartan efficacy in reducing the activity of matrix metalloproteinase-9, inducing activity of its natural inhibitor TIMP-3, paralleled with improved left ventricle hemodynamics observed in post- myocardial infarction remodeling should be tested to prevent pacing induced heart remodeling. Such a study is already to start [25]. The trial is powered to detect the $5 \%$ point difference in left ventricle ejection fraction over the 12 months. Secondary measures of echocardiographic assessment, MMP-9 and TIMP-3 plasma activity, functional capacity of participants, as well as safety issues will also be monitored throughout the study.

If valsartan proves effective, it will be an attractive measure to improve long term prognosis in aging population and increasing number of pacemaker recipients.

\section{Acknowledgements}

AT is supported by Medical University of Silesia grant No KNW$1-191 / \mathrm{K} / 5 / 0$.

\section{References}

1. Chiladakis JA, Koutsogiannis N, Kalogeropoulos A, Zagli F, Arvanitis P, et al. (2007) Unfavourable effects of continuous, atrial-synchronised ventricula pacing on ventricular systolic and diastolic function in patients with normal left ventricular ejection fraction: usefulness of tissue and colour Doppler echocardiography. Hellenic J Cardiol 48: 335-340.

2. Thambo JB, Bordachar P, Garrigue S, Lafitte S, Sanders P, et al. (2004) Detrimental ventricular remodeling in patients with congenital complete heart block and chronic right ventricular apical pacing. Circulation 110: 3766-3772.

3. Tops LF, Suffoletto MS, Bleeker GB, Boersma E, van der Wall EE, et al. (2007) Speckle-tracking radial strain reveals left ventricular dyssynchrony in patients with permanent right ventricular pacing. J Am Coll Cardiol 50: 1180-1188.

4. Sá LA, Rassi S, Batista MA (2013) Is conventional cardiac pacing harmful in patients with normal ventricular function? Arq Bras Cardiol 101: 545-553.

5. Zhang X-H, Chen HUA, Siu C-W, Yiu K-H, Chan W-S, et al. (2008) New-Onse Heart Failure After Permanent Right Ventricular Apical Pacing in Patients with Acquired High-Grade Atrioventricular Block and Normal Left Ventricular Function. J Cardiovasc Electrophysiol 19: 136-141.

6. García RA, Brown KL, Pavelec RS, Go KV, Covell JW, et al. (2005) Abnorma cardiac wall motion and early matrix metalloproteinase activity. Am J Physio Heart Circ Physiol 288: H1080-1087.

7. Lin JM, Lai LP, Lin CS, Chou NK, Chiu CY, et al. (2010) Left ventricula extracellular matrix remodeling in dogs with right ventricular apical pacing. $J$ Cardiovasc Electrophysiol 21: 1142-1149.

8. Tse HF, Yu C, Wong KK, Tsang V, Leung YL, et al. (2002) Functional abnormalities in patients with permanent right ventricular pacing: the effect of sites of electrical stimulation. J Am Coll Cardiol 40: 1451-1458.

9. Sato I, Shimada K (2001) Quantitative analysis of tenascin in chordae tendineae of human left ventricular papillary muscle with aging. Ann Anat 183: 443-448.

10. Nishioka T, Onishi K, Shimojo N, Nagano Y, Matsusaka H, et al. (2010) Tenascin-C may aggravate left ventricular remodeling and function after myocardial infarction in mice. Am J Physiol Heart Circ Physiol 298: H10721078.

11. Imanaka-Yoshida K, Hiroe M, Nishikawa T, Ishiyama S, Shimojo T, et al. (2001) Tenascin-C modulates adhesion of cardiomyocytes to extracellular matrix during tissue remodeling after myocardial infarction. Lab Invest 81: 1015-1024.

12. Nyberg P, Xie L, Kalluri R (2005) Endogenous inhibitors of angiogenesis Cancer Res 65: 3967-3979.

13. Mundel TM, Kalluri R (2007) Type IV collagen-derived angiogenesis inhibitors. Microvasc Res 74: 85-89.

14. Hamano $Y$, Zeisberg M, Sugimoto H, Lively JC, Maeshima Y, et al. (2003) Physiological levels of tumstatin, a fragment of collagen IV alpha3 chain, are generated by MMP-9 proteolysis and suppress angiogenesis via alphaV beta3 integrin. Cancer cell 3: 589-601. 
Citation: Tomasik A (2015) Perspectives on Pharmacological Prevention of Pacing Induced Heart Remodeling: Targeting Extracellular Matrix. Single Cell Biol 4: 120. doi:10.4172/2168-9431.1000120

Page 3 of 3

15. Spinale FG1 (2007) Myocardial matrix remodeling and the matrix metalloproteinases: influence on cardiac form and function. Physiol Rev 87: 1285-1342.

16. Whittaker M, Floyd CD, Brown P, Gearing AJ (2001) Design and therapeutic application of matrix metalloproteinase inhibitors. (Chem. Rev. 1999, 99, 27352776. Published on the web september 8, 1999). Chem Rev 101: 2205-2206.

17. Chen J, Chu Y, Cao J, Wang W, Liu J, et al. (2011) Effects of T-2 toxin and selenium on chondrocyte expression of matrix metalloproteinases (MMP-1, MMP-13), $\alpha 2-m a c r o g l o b u l i n(\alpha 2 M)$ and TIMPs. Toxicol In Vitro 25: 492-499.

18. Maquoi E, Sounni NE, Devy L, Olivier F, Frankenne F, et al. (2004) AntiInvasive, Antitumoral, and Antiangiogenic Efficacy of a Pyrimidine-,4,6-trione Derivative, an Orally Active and Selective Matrix Metalloproteinases Inhibitor. Clin Cancer Res 10: 4038-4047.

19. Yan YL, Miller MT, Cao Y, Cohen SM (2009) Synthesis of hydroxypyrone- and hydroxythiopyrone-based matrix metalloproteinase inhibitors: Developing a structure-activity relationship. Bioorg Med Chem Lett 19: 1970-1976.

20. Zakeri B, Wright GD (2008) Chemical biology of tetracycline antibiotics. Biochem Cell Biol 86: 124-136.
21. Hudson MP, Armstrong PW, Ruzyllo W, Brum J, Cusmano L, et al. (2006) Effects of Selective Matrix Metalloproteinase Inhibitor (PG-116800) to Prevent Ventricular Remodeling After Myocardial Infarction: Results of the PREMIER (Prevention of Myocardial Infarction Early Remodeling) Trial. J Am Coll Cardiol 48: 15-20.

22. Spinale FG, Coker ML, Krombach SR, Mukherjee R, Hallak H, et al. (1999) Matrix metalloproteinase inhibition during the development of congestive heart failure: effects on left ventricular dimensions and function. Circ Res 85: 364-376.

23. Sawicki G, Menon V, Jugdutt BI (2004) Improved balance between TIMP-3 and MMP-9 after regional myocardial ischemia-reperfusion during AT1 receptor blockade. J Card Fail 10: 442-449.

24. Miyazaki S, Kasai T, Miyauchi K, Miyazaki T, Akimoto Y, et al. (2010) Changes of matrix metalloproteinase- 9 level is associated with left ventricular remodeling following acute myocardial infarction among patients treated with trandolapril, valsartan or both. Circ J 74: 1158-1164.

25. Tomasik A, Jachec W, Wojciechowska C, Kawecki D, Bialkowska B, et al (2015) Randomized placebo controlled blinded study to assess valsartan efficacy in preventing left ventricle remodeling in patients with dual chamber pacemaker - Rationale and design of the trial. Contemp Clin Trials 42: 239-243. 